Chapter 8

\title{
Patient on ACS Pathway - Hypomagnesaemia a Contributory Factor to Myocardial Ischemia
}

\author{
Ghulam Naroo, Tanveer Ahmed Yadgir, \\ Bina Nasim and Omer Skaf \\ Additional information is available at the end of the chapter \\ http://dx.doi.org/10.5772/55286
}

\section{Introduction}

Magnesium is the $4^{\text {th }}$ most abundant intracellular cation in the body. Normal adult plasma concentration ranges from 1.7 to $2.5 \mathrm{mg} / \mathrm{dL}$. Most of the body's reserves are found in the skeletal bone mass.

Hypomagnesaemia is a common electrolyte abnormality seen in around $12 \%$ hospitalized patients and has an incidence as high as 60 to $65 \%$ in Intensive Care Unit patients.

Clinical signs and symptoms are only possible in severe magnesium deficiency. Surprisingly, magnesium depletion can present despite a near normal serum magnesium level.

Common nutritional sources include green leafy vegetables, legumes, nuts, animal proteins, seafood and sea greens like kelp.

Absorption takes place in the upper small intestine, where nearly 30 to $50 \%$ of consumed magnesium is taken up depending upon the endogenous magnesium status.

Magnesium is excreted by the kidneys.

In circulation $33 \%$ is albumin bound (non-filterable), $12 \%$ complexed with anions \& $55 \%$ is in the free ionized form (filterable fraction) 


\section{Magnesium at cellular level}

Magnesium serves as a cofactor for over 300 enzymes involved in Deoxyribonucleic Acid (DNA) and Ribonucleic Acid (RNA) synthesis, protein synthesis, energy metabolism and maintenance of electrical potential of nervous tissue and cell membranes.

Of particular importance is the role of this element in regulating potassium fluxes through Sodium (Na) Pottasium (K) ATPase pump and involvement in metabolism of Calcium. (Magnesium is a natural calcium channel blocker)

Na K Pump - the sodium potassium pump is activated by magnesium. With magnesium deficiency there is impaired pump activity, whereby insufficient potassium can be pumped into the cell, although the supply may be great enough.

The energy substrate for the transport activity of the sodium/potassium pump is represented by Adenosine Triphosphate (ATP) in form of its magnesium complex. This ATP- $\mathrm{Mg}^{++}$complex is split by the ATPase delivering the transport energy and therefore it is said that the ATPase is directing the sodium/potassium pump.

Furthermore with Magnesium deficiency there is not enough energy substrate. Hence the cell membrane shows increased permeability and the potassium gradient cannot be maintained.

Potassium leaves the cell, in compensation sodium and hydrogen influx takes place passively. Magnesium leaves the cell, if not enough ATP is present for forming the ATP-Mg complex and calcium influx will follow.

Calcium (Ca) Pump and Na/Ca exchange - There are two possibilities for elimination of calcium out of the cell, but unfortunately both are impaired by magnesium deficiency:

They are the calcium pump and the sodium/calcium exchange.

Calcium pump- After muscle contraction calcium ions will be transported back again from the cytosol to the stores of the sarcoplasmatic reticulum by the calcium pump. The concentration gradient at this action needs a high expense of energy: one ATP for two calcium ions. The calcium transport-ATPase is magnesium dependent.

Sodium calcium exchange-. During the action potential calcium influx takes place along the slow calcium channels into the cell and induces the contraction process. The calcium influx will be compensated again by an exchange of three sodium ions into the cell. The energy for the exchange originates from the high extracellular sodium concentration, but these three sodium-ions must be removed again out of the cell by the sodium/potassium pump which requires one ATP. If the performance of the sodium/potassium pump is impaired, cellular sodium will increase and inhibit sodium/calcium exchange. This can be due to ATP deficiency, myocardial ischemia/ reperfusion injury, potassium/ magnesium deficiency.

Increased sodium within the cell results in hypertension and theincreased calcium within the cell increases the vascular tone in the smooth muscle of the artery to aggravate the hypertension. 


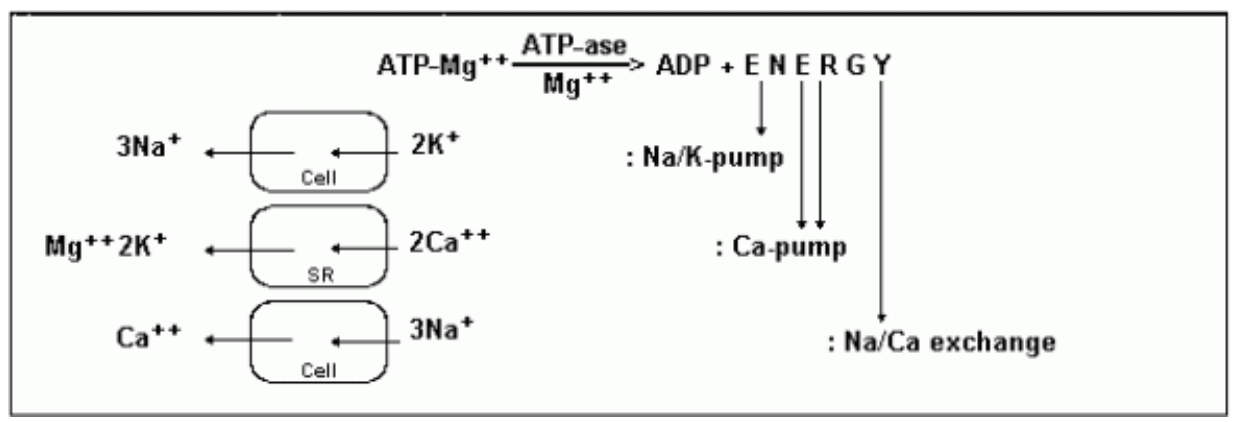

Figure 1. ATP-Mg++ : Energy Supply for pumps and exchange

\section{Causes of hypomagnesaemia}

Pathologic effects of primary nutritional deficiency of magnesium is rare unless a relatively low magnesium intake is accompanied with prolonged diarrhea or excessive urinary magnesium losses.

Gastrointestinal Causes-

- Acute/ Chronic Diarrhea

- Malabsorption syndromes

- Steatorrhea

- Small bowel bypass

- Inborn errors of metabolism - autosomal recessive disorder, chromosome 9, selective defect in magnesium absorption due to a mutation in gene that encodes for a member of receptor channel family.

- Acute pancreatitis - Saponification of magnesium and potassium ions.

Renal Causes-

- Loop and thiazide diuretics - cause mild hypomagnesaemia, because volume contraction increase proximal sodium, water and magnesium reabsorption

- Alcoholics- Alcohol induced tubular dysfunction which is reversible within 4 weeks.

- Hypercalcemia- increased filtered calcium load competes with magnesium in transport across the ascending limb of loop of Henle.

- Nephrotoxins- Aminoglycosides, amphotericin B, cisplatin, cyclosporine

- Loop of Henle \& Distal tubule dysfunction - Barters Syndrome and Gitelmans syndrome. 


\section{Effects of hypomagnesaemia on tissues}

- Hypokalemia- Hypokalemia induced due to deficiency of magnesium is refractory to potassium supplementation until magnesium deficiency is corrected.

- Hypocalcaemia- due to diminished secretion of Parathyroid hormone and resistance to the effect of parathyroid hormone at the receptor level. Parathyroid hormone (PTH) induced release of calcium from bone is impaired when plasma $\mathrm{Mg}<0.8 \mathrm{mg} / \mathrm{dL}$.

- Vitamin D Deficiency- Low plasma level of Calcitriol is noted in Hypomagnesaemia which further contributes to Hypocalcaemia.

\section{Clinical manifestations of hypomagnesaemia}

- Cardiac Effects - Arrhythmias, Heart Disease

- Metabolic Effects - Hypokalemia, Hypocalcemia, Diabetes, Osteoporosis

- Neurological Effects- Headache, paresthesias, tremors, muscular spasms, tetany, convulsions, migraine, irritability, anxiety, weakness, mood swings, depression.

- ECG Changes-

Initial stage- widening of QRS Complex peaking of T waves

Later stage- prolongation of PR interval, progressive widening of QRS complex and diminution of T wave.

\section{Diagnosis of magnesium deficiency}

The serum magnesium level correlates poorly with total body stores. As a result, there have been several intracellular assays of magnesium from lymphocytes, red blood cells, and muscle biopsies. These assays include nuclear magnetic resonance (NMR) spectroscopy and ionspecific electrode measures. But since these tests are very expensive, therefore they are not clinically applicable at present. For these reasons, despite its limitations serum magnesium levels are commonly and easily carried out to evaluate the magnesium status. If the serum magnesium level is low, intracellular magnesium is also low but it is important to understand that many patients may have normal serum magnesium levels but may still be intracellularly depleted. [1]

\section{Discussion}

Low serum magnesium is an independent predictor of CHD in both gender. Although relation exist more in women and less in men. A cross-sectional cohort study has shown inverse 
association between serum magnesium and carotid intima-media thickness(12.). Low serum magnesium causes endothelial damage that accelerates the atherosclerotic process leading to ACS. Evidence links significant low serum magnesium to CHD in patient with Acute Myocardial Infarction (AMI) versus control [13] [14]. This cohort of 15,792 middle aged subjects were assessed over a four to seven year period as part of the Atherosclerosis Risk in Communities (ARIC) study [2]. The relative risk of CHD across quartiles of serum magnesium was 1.0 (in the lowest quartile), $0.92,0.48$ and 0.44 . Both men and women who developed CHD had lower mean baseline serum magnesium concentration than the disease-free controls.

Another study of 50 patients with coronary heart disease found that oral magnesium supplement therapy improved endothelial function and exercise tolerance compared to placebo. Autopsy has shown that magnesium concentrations in cardiac muscle of individuals who died of heart disease is lower than those of accident victims [15] [16] One study, found a $20 \%$ reduction in magnesium in the non-infarcted and a 50\% reduction in the infarcted myocardium. Also infarcted myocardium had a depressed magnesium/calcium ratio [16]. A prospective National Health and Nutritional Examination Survey (NHANES) I follow up study showed the important role of modifiable dietary and behavioral characteristics in the causation and prevention of coronary heart disease hospitalization and mortality. This study was done over a 10 years follow up of 8251 subjects. The study emphasized the role of modifiable dietary factors including magnesium as well as behavioral characteristics in the causation and prevention of CHD [17].

Another epidemiologic study showed an inverse association between dietary magnesium and incident CHD. These associations were present after adjustment for multiple confounding factors, including race, smoking, alcohol intake, life style \& exercise, waist/hip ratio, fibrinogen \& lipids level, diuretics use and HRT [17]. Diabetes and hypertension may merely be confounders but low magnesium concentration may contribute to the pathogenesis of diabetes or hypertension [18] [19]. High intake of foods rich in magnesium including green vegetables, nuts and whole grains may provide protection against CHD by increasing magnesium levels [20]

The following effects of magnesium might play an important role in protecting the myocardium in patients of Unstable Angina:

- Reducing the ischemic myocardial death by reducing the intracellular calcium overload by limiting the reperfusion injury.

- Dilating the coronary arteries. [21]

- Reducing the after load. [22]

- Inhibiting the platelet function by its effect on prostacyclin secretion.

- Attenuating the catecholamine release by reducing the sympathetic activity. [23]

Other effects of magnesium beneficial in myocardial infarction and life threatening ventricular arrhythmias include the above and following:

- Direct antiarrhythmic effect. 
- Reducing free radical formation.

- Enhanced collateral flow.

Magnesium has been shown to inhibit calcium influx in the cell, it reduces the mitochondrial calcium overload, conserves the intracellular ATP as Mg2+-ATP, and raising extracellular magnesium has been shown to be protective in ischemia. Magnesium inhibits the spasm of the coronary arteries [21], increases the coronary blood flow, and decreases the coronary vascular resistance in patients of Variant Angina. This can be a direct effect of magnesium on coronary vasculature or it can be an indirect effect by reducing the catecholamine release.

High catecholamine levels play an important role in the pathogenesis and prognosis of unstable angina and are closely related to the extension of an infarct [24]. Magnesium tends to inhibit the release of catecholamines from the adrenal medulla and reduces the sensitivity of a- adrenergic receptors to catecholamines, thereby reducing the arrythmogenic and the pressor effects of the catecholamines [25]. Deficiency of magnesium therefore enhances the sympathetic activity and increases the catecholamine induced myocardial damage.

A double blind randomized placebo controlled study was conducted to assess the 24 hour infusion of magnesium in patients of unstable angina [26]. In this study the patients who presented with unstable angina and had electrocardiographic changes were randomized to receive 24 hour intravenous infusion of magnesium or placebo within $12 \mathrm{hr}$ of admission. The chosen primary endpoints in this study included ECG changes as assessed by $48 \mathrm{~h}$ Holter monitoring, resting 12 lead ECGs, CK-MB release and urinary catecholamine levels.

In this study patients were followed for 1 month. Thirty-one patients received magnesium sulphate and 31 placebo. Baseline characteristics and extent of coronary disease were similar in both groups. On $48 \mathrm{~h}$ Holter monitoring, 14 patients(50\%) were found to have transient ST segment shifts in the magnesium group versus 12 patients $(46 \%)$ in the placebo group. However, there were fewer ischemic episodes in the magnesium group (51 versus $101, \mathrm{P}<0001$ ) and there was a trend towards an increase in the total duration of ischemia in the placebo group compared to the magnesium group in the second $24 \mathrm{~h}$. It was found that regression of $\mathrm{T}$ wave changes on the $24 \mathrm{~h}$ ECG and reduction in the ST segment changes in the 12 lead ECG, occurred more frequently in patients who received magnesium compared to those treated with placebo (11 patients versus 0 patients respectively, $\mathrm{P}<0005$ ).

Creatine kinase-MB release was significantly less at 6 and $24 \mathrm{~h}$ in patients who received magnesium compared to those treated with placebo. Catecholamine excretion was lower in patients treated with magnesium than in those treated with placebo in the first $12 \mathrm{~h}$ sample, $P<005)$. On continuous ECG monitoring, a similar proportion of patients in each treatment group had evidence of myocardial ischemia in the first $24 \mathrm{~h}$ of recording. However, the number of episodes was significantly less in the magnesium group and the number of patients with transient myocardial ischemia in this group fell from 11 patients $(39 \%)$ to five $(18 \%)$ in the second $24 \mathrm{~h}$ of recording with no change in the placebo group (10 patients [39\%] in both the first and second $24 \mathrm{~h}$ ). 
This study concluded that magnesium infusion reduces the ischemic ECG changes, cardiac markers, and urinary catecholamine excretion in the acute phase of unstable angina. Therefore magnesium is useful in these patients.

Among the other studies which have suggested that magnesium may reduce mortality and serious arrhythmias post acute myocardial infarction is the Second Leicester intravenous magnesium intervention trial (LIMIT-2) study [27], which was a double-blind randomized trial of 2316 patients with suspected acute MI who received either intravenous magnesium sulfate or placebo along with other currently accepted therapies for MI, including thrombolysis. Thirty-five percent of the patients received a fibrinolytic agent, usually streptokinase, and 66 percent received aspirin. The presence of an acute MI was confirmed in 65 percent of cases.

An important design feature of this trial was that magnesium was administered prior to a fibrinolytic agent. A treatment effect was observed in all subgroups, including those receiving thrombolysis and it showed a $24 \%$ reduction in mortality, $25 \%$ reduction in the incidence of left ventricular failure and $21 \%$ reduction in the mortality from ischemic heart disease.

In another small trial which revealed a positive association of magnesium to coronary heart disease, 194 patients with an acute MI who were not considered candidates for fibrinolytic therapy were randomized to receive either intravenous magnesium sulfate or placebo. In this study the benefits of magnesium compared to placebo showed a reduction in the in-hospital mortality especially in the elderly and a lower incidence of both arrhythmias and left ventricular dysfunction.

However, this was not supported by the results of International Study of Infarct Survival (ISIS-4) study [28] which showed that a 24-h infusion of magnesium has no beneficial effect in those receiving thrombolysis for acute myocardial infarction. In this study, patients were randomized after the thrombolytic agent had been administered, a mean of $8 \mathrm{~h}$ after the onset of pain, compared to a median of $3 \mathrm{~h}$ in the Leicester intravenous magnesium intervention trial (LIMIT-2) trial, in which iv magnesium was given prior to thrombolysis. Animal studies have shown that the effect of magnesium is greatest if given before spontaneous or induced reperfusion, as this reduces the reperfusion induced myocardial injury, and this may account, in part, for the lack of benefit seen in International Study of Infarct Survival (ISIS-4).

\section{Conclusion}

Data suggest that hypomagnesemia may precede CHD. The U.S. National Academy of Sciences has estimated that a nation-wide initiative to add calcium and magnesium to soft water might reduce the annual cardiovascular death rate by 150,000 in the United States. [7]. It is recommended to design further observational and interventional studies to substantiate the link. 


\section{Author details}

Ghulam Naroo ${ }^{1}$, Tanveer Ahmed Yadgir ${ }^{2}$, Bina Nasim ${ }^{3}$ and Omer Skaf ${ }^{4}$

1 Emergency \& Trauma Centre, Rashid Hospital Dubai, United Arab Emirates

2 Research \& Accreditation Department, Dubai Corporation for Ambulance Services, Dubai, United Arab Emirates

3 Rashid Hospital, Dubai, United Arab Emirates

4 Dubai Corporation for Ambulance Services, Dubai, United Arab Emirates

\section{References}

[1] Altura, B. M, Brodsky, M. A, Elin, R. J, et al. Magnesium: growing in clinical importance. Patient Care (1994). , 10, 130-150.

[2] Liao, F, Folsom, A. R, \& Brancati, F. L. Is low magnesium concentration a risk factor for coronary heart disease? The Atherosclerosis Risk in Communities Study. Am Heart J (1998).

[3] Taneva, E. Hypokaliaemia and hypomagnesemia during acute coronary syndrome: A- 661. European Journal of Anaesthesiology (2005). , 22-issue, 172.

[4] Altura, B. M. Aimin Z Altura BT: Magnesium, hypertensive vascular disease, atherogenesis, subcellular compartmentation of calcium and magnesium and vascular contractility. Miner Electrolyte Metab (1993). , 19, 323-336.

[5] Paolisi, G. Barbagallo M: Hypertension, diabetes, and insulin resistance: the role of intercellular magnesium. Am J Hypertension (1997). , 10, 346-355.

[6] Chester Fox MD, Delano Ramsoomair, MD, and Cathleen Carter, PhD: Magnesium: Its Proven and Potential Clinical Significance. South Med J. (2001).

[7] http:/ / www.mgwater.com/, The Magnesium website.(2002). access dated- 27/11/12)

[8] Oral Mg therapy improves endothelial function in pt with ACSShechter M,Sharir Circulation.(2000).

[9] The important role of modifiable dietary and behavioral characterisics in the causation and prevention of coronary heart disease hospitalization and mortality; the perspective NHANES follow up study, Gartside PS, Glueck CJ Jam Coll Nutr.(1995).

[10] 10. Antman, EM, Anbe, DT, \& Armstrong, PW. ,et al. ACC/AHA guidelines for the management of patients with ST-elevation myocardial infarction. 
[11] Redwood, S. R, Basir, Y, Huang, J, et al. Effect of magnesium sulphate in patients with unstable angina. A double blind,randomized, placebo-controlled study.Eu Heart J (1997).

[12] Ma, J, Folsom, A. R, Melnick, S. L, Eckfeldt, J. H, Sharrett, A. R, Nabulsi, A. A, et al. Associations of serum and dietary magnesium with cardiovascular disease, hypertension, diabetes, insulin, and carotid arterial wall thickness: the ARIC Study. J Clin Epidemiol (1995). , 48, 927-40.

[13] Singh, R. B, Rastogi, S. S, Ghosh, S, \& Niaz, M. A. Dietary and serum magnesium levels in patients with acute myocardial infarction, coronary artery disease and noncardiac diagnoses. J Am Coll Nutr (1994). , 13, 139-43.

[14] Kafka, H, Langevin, L, \& Armstrong, P. W. Serum magnesium and potassium in acute myocardial infarction: influence on ventricular arrhythmias. Arch Intern Med (1987). , 147, 465-9.

[15] Marier, J. R. Water hardness, human health and the importance of magnesium. Ottawa: National Research Council of Canada. NRCC Series (1979). (17581), 65-84.

[16] Speich, M, Bousquet, B, \& Nicolas, G. Concentrations of magnesium, calcium, potassium, and sodium in human heart muscle after acute myocardial infarction. Clin Chem (1980). , 26, 1662-5.

[17] The role of modifiable dietary and behavioral characteristics in the causation and prevention of coronary heart disease hospitalization and mortality- NHANES follow up study-1.

[18] White JR Jr. Campbell RK. Magnesium and diabetes: a review. Ann Pharmacother (1993). , 27, 775-80.

[19] 31. Resnick, L. M. Cellular calcium and magnesium metabolism in the pathophysiology and treatment of hypertension and related metabolic disorders. Am J Med (1992). A):11S-20S.

[20] 48. Fraser, G. E, Sabate, J, Beeson, W. L, \& Strahan, T. M. A possible protective effect of nut consumption on risk of coronary heart disease: the Adventist Health Study. Arch Intern Med (1992). , 152, 1416-24.

[21] Vigorito, C, Giordano, A, Ferraro, P, et al. Hemodynamic effects of magnesium sulfate on the normal human heart. AmJ Cardiol (1991). , 67, 1435-7.

[22] Rasmussen, H, Larsen, O, Meier, K, \& Larsen, J. Hemodynamic effects of intravenously administered magnesium on patients with ischemic heart disease. Clin Cardiol (1988). , 824-8.

[23] James, M, Cork, R, Harlen, G, \& White, J. Interactions of adrenaline and magnesium on the cardiovascular system of the baboon. Magnesium (1988). 
[24] Penny, W. J. The deleterious effects of myocardial catecholamines on cellular electrophysiology and arrhythmias during ischaemia and reperfusion. Eur Heart J (1984). , 5, 960-73.

[25] Bean, B, \& Varghese, P. Role of dietary magnesium deficiency in the pressor and arrhythmogenic response to epinephrine in the intact dog. Am Heart J (1994). , 127, 96-102.

[26] Redwood Sr, Bashir Y, Huang J. Effect of magnesium sulfate in patients of unstable angina. European heart journal (1997).

[27] Woods Kl, Flether S. Long term outcome after intravenous magnesium sulfate in suspected acute myocardial infarction. The second Leicester intravenous magnesium intervention trial. LIMIT-2. Lancet (1994).

[28] ISIS-4the Fourth International Study of Infarct Survival- Lancet (1995). 